\title{
Multimodal Presentation of Local Danger Warnings for Drivers: A Situation-dependent Assessment of Usability
}

\author{
Yujia Cao \\ Human Media Interaction \\ University of Twente \\ Enschede, the Netherlands \\ y.cao@utwente.nl
}

\author{
Mariët Theune \\ Human Media Interaction \\ University of Twente \\ Enschede, the Netherlands \\ m.theune@utwente.nl
}

\author{
Christian Müller \\ German Research Center \\ for Artificial Intelligence \\ Campus Saarbrücken, \\ Germany \\ christian.mueller@dfki.de
}

\begin{abstract}
This study addresses how advanced driver assistance systems (ADAS) should communicate with drivers, focusing on the local danger warning function. To achieve high-quality assistance, the communication mode needs to be adaptive to changes in driving situation (driver's state, workload and environment). In a user study investigating different warning communication modes, drivers were required to assess the usability of each communication mode in five different driving situations. Results revealed that the driving situation significantly affected the perceived usefulness of each warning communication mode, mainly due to the use of modality. Moreover, regardless of communication mode, the local danger warning function was considered as the most useful in the low visibility situation and the least useful in the highly demanding driving situation. Findings of this study can be applied to the design of ADAS in general. Keywords: multimodal presentation, local danger warning, adaptability.
\end{abstract}

\section{Introduction}

Advanced Driver Assistance Systems (ADAS) are incar systems designed to reduce or eliminate driver's error, and enhance the efficiency of traffic [1]. One important function of ADAS, among others, is local danger warning. Local danger warning aims to extend the driver's horizon and warn him/her of dangerous situations coming up. Recent advances in inter-vehicle communication technology have largely promoted the application of local danger warnings, because relevant information can be shared between cars at runtime [2].

To achieve high-quality assistance, ADAS need to communicate with the driver in an effective and efficient way. This is especially true in case of local danger warnings; because first, they are usually low-frequency events, so drivers might be less ready for them compared with other ADAS functions. Second, they are highly urgent, so drivers usually have very limited time to think and react. Therefore, local danger warning messages should be communicated in a way that allows them to be picked up quickly (efficiency) and correctly (effectiveness).

A challenge to reach this goal lies in the fact that driving conditions are very diverse, such as in heavy traffic, on an empty highway, under strong sunlight, in the night, in the fog, just to name a few. Variations in driving conditions alter the demand driving imposes on the driver, as well as the requirements on appropriate communication modes. For example, auditory messages are appropriate in a low-visibility condition, because drivers need to keep their eyes on the road. However, they might be less effective when there are rich sounds in the car, such as radio and conversations. Therefore, there might not be one fixed communication mode that is the optimum for all conditions. Instead, ADAS need to be adaptive to changes in the needs of the user, his/her workload and the environment that (s)he is operating in $[3,4]$.

In this study, we investigated the usability of various communication modes for local danger warnings. Two aspects were considered, namely the level of assistance (what to communicate) and the use of modality (how to communicate). Besides the influence of communication modes on the effectiveness and efficiency of warnings, we also intended to investigate the expected usefulness of each communication mode in different driving situations. To this end, drivers were required to perform a situationdependent assessment for each mode, based on their reallife experiences. The selection of situation took into account both environmental and cognitive factors. This subjective assessment can be considered as a very first step in the design process of a fully adaptive system, because the results provide understating of drivers' needs in different situations and how they expect the system to adapt.

The remainder of the paper is organized as follows. First, the experiment is described, including task, warning design, procedure and measurements. We then present and discuss the results. Finally, conclusions and future directions are given. 


\section{Experiment}

A user study was carried out using a driving simulator integrated in a real car. Drivers drove on a highway with two lanes for the same direction. At random intervals, they received warnings about road obstacles in a short distance ahead (8 to 10 seconds' drive) but not yet visible. A warning message contained three elements: obstacle type, location and distance. To avoid the danger, drivers were required to change lane if the obstacle was on the nearside lane and to brake if the obstacle was on the offside lane or on the roadside.

The warning communication mode was manipulated by two factors. The level of assistance varied between warning only and warning preceded by action suggestion (brake or lane change). The use of modality had four options: speech warning, visual warning with beep sound cue, visual warning with blinking bar cue, and combined visual and speech warning. Note that action suggestions were always communicated via speech. In total, eight communication modes were investigated, as summarized in Table 1.

Table 1. The eight communication modes investigated.

\begin{tabular}{|l|c|c|c|c|c|c|c|c|}
\hline & 1 & 2 & 3 & 4 & 5 & 6 & 7 & 8 \\
\hline Action suggestion & & & & & $\sqrt{ }$ & $\sqrt{ }$ & $\sqrt{ }$ & $\sqrt{ }$ \\
\hline Visual message & $\sqrt{ }$ & $\sqrt{ }$ & $\sqrt{ }$ & & $\sqrt{ }$ & $\sqrt{ }$ & $\sqrt{ }$ & \\
\hline Speech message & $\sqrt{ }$ & & & $\sqrt{ }$ & $\sqrt{ }$ & & & $\sqrt{ }$ \\
\hline Beep sound cue & & $\sqrt{ }$ & & & & $\sqrt{ }$ & & \\
\hline Blinking bar cue & & & $\sqrt{ }$ & & & & $\sqrt{ }$ & \\
\hline
\end{tabular}

Thirty-two drivers participated in the experiment. The procedure started with an introduction, followed by a training session. Afterwards, each driver drove eight tracks with warnings communicated in different modes. The track order was counter-balanced. Drivers were required to fill in questionnaires during the short breaks between two tracks.

Following the ISO usability standard (ISO 9241-11 [5]), we evaluated the warning communication modes in terms of effectiveness (danger avoidance performance, message recall performance, etc.), efficiency (reaction time, driving load assessment, etc.) and satisfaction (situation-dependent usability assessment). Regarding effectiveness and efficiency, a detailed description of the measurements and the results can be found in [6]. Here, we focus on satisfaction.

Satisfaction on warning communication was measured in terms of situation-dependent subjective assessments. Drivers were asked to evaluate how useful each communication mode would be in five specified situations:

1. Rich sound - when driving with rich surrounding sounds (noise, radio, conversation, etc.)

2. Low visibility - when driving with a low visibility (in the night, fog, etc.)
3. Fatigue - when being tired and unconcentrated

4. Long drive - during a long and boring drive (e.g., a long trip on the highway)

5. High demand - when driving in highly demanding situations (in heavy traffic, in an unfamiliar city, etc.)

Each rating was performed on a 6-level scale from 0 (not useful at all) to 5 (very useful). Note that these situations were not actually simulated in the experiment, thus the ratings reflect the expected usefulness based on drivers' real-life experiences. By analyzing the ratings, we intended to answer the following two questions:

- In which situation(s) is the assistance of local danger warning thought to be more useful (appreciated)?

- In each driving situation, which communication modes make the warnings more useful?

\section{Results}

First, a two-way repeated-measure ANOVA was conducted on the rating scores, using warning communication mode (simply referred to as 'mode' in this section) and situation as two independent factors. Results showed that both factors had a significant influence on the usefulness of warnings (mode: $F(7,25)=11.7, p<0.001$; situation: $F(4,28)=24.1, p<0.001)$. As expected, there was also an interaction effect between these two factors $(F(28,4)=5.7, p<0.05)$.

In the 'low visibility' situation, the modes received the highest usefulness rating score in average, whereas in the 'high demand' situation they received the lowest (see Figure 1). Post-hoc analysis (Bonferroni tests) further revealed significant differences in rating score between 'low visibility' and each of the other four situations. The same was also found for the 'high demand' situation. In combination, these results suggest that the assistance of local danger warnings could be generally more useful and more needed (appreciated) in some situations than in others, regardless of how warnings are communicated. Drivers in this study considered local danger warnings the most useful when the visibility was low and the least useful when driving itself was highly demanding.

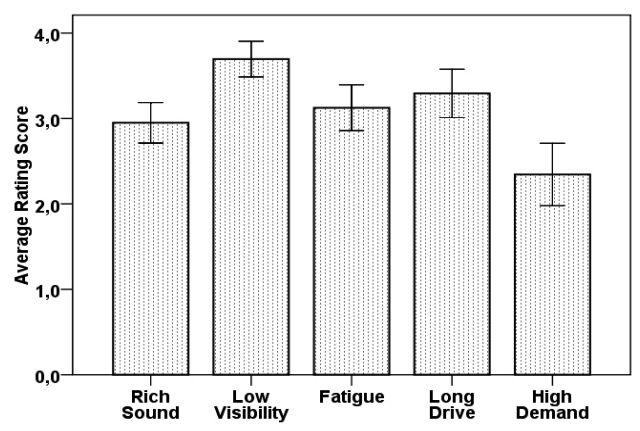

Figure 1. Average rating score over all modes for each situation. Error bars represent standard errors. 
We then moved on to investigating which mode(s) made the warnings the most useful in each driving situation. The approach was to zoom into each situation and perform a two-way repeated-measure ANOVA on the rating scores, using modality and level of assistance as independent factors.

The results regarding the level of assistance showed a high consistency - in all five situations, warnings with action suggestions (AS) were considered significantly more useful than warnings without AS (see Figure 2). This suggests that AS could be generally beneficial, regardless of the use of modality and the driving situation. However, results from the modality factor revealed diversity, which actually explained the interaction effect between mode and situation.

Rich sound: when there are rich sounds in the driving environment, visual modalities are expected to be highly necessary, because the saliency of auditory modalities degrades in proportion to competing surrounding sound level [7]. Indeed, the two purely auditory presentation modes (speech with and without AS) were rated as the least useful (see Figure 2(a)). Post-hoc (Bonferroni) tests confirmed that speech (only) received significantly lower rating scores than each of the other three modality variants. The difference between the other three modality variants did not reach a statistically significant level, indicating that as long as there are visual modalities involved, the warning communication is useful in this driving condition.

Low visibility: in this condition the 'speech + visual' modality variant was considered as the most useful (see Figure 2(b)). It was shown by Bonferroni tests to be significantly more useful than each of the other three variants. The explanation of this finding is twofold. First, when visibility is low, it is particularly important to keep one's eyes on the road. Therefore, it should be more appropriate to communicate warnings orally rather than visually. Second, using only speech does not offer cognitive advantages because speech does not allow free perception [7], meaning that attention has to be focused on the speech during its presentation, in order to fully perceive the content. Therefore, it is beneficial to also provide visual warnings as supplement to speech warnings.

Fatigue: in this condition, the 'speech + visual' and 'beep + visual' variants were rated as significantly more useful than the other two modality variants, and there was no significant difference between the two (Figure 2(c)). This finding can be explained by the fact that auditory modalities are much more salient than visual modalities. Attention is promptly directed to an auditory signal upon the onset of its presentation [8]. When drivers are tired and unconcentrated, they tend to be less attentive. In this case, the speech warning and the beep sound cue were both considered useful, because they are able to attract attention timely and increase vigilance level. However, using a purely auditory modality (speech only) was still not preferred. Furthermore, an interaction effect between the modality and the level of assistance was also found. This is because the benefit of AS (always in speech) was particularly pronounced with the 'blink + visual' modality variant, which was purely visual without AS.

(a)

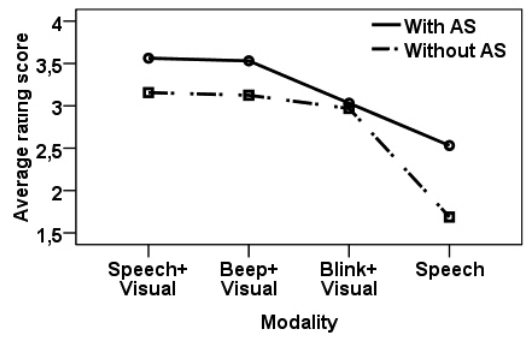

(b)

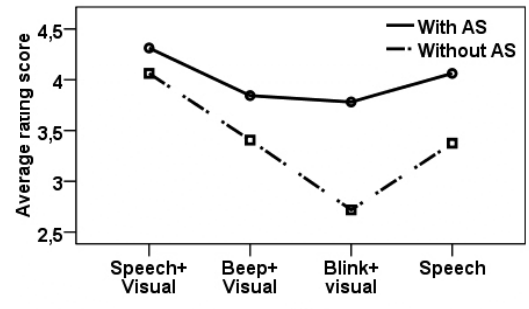

Modality

(c)

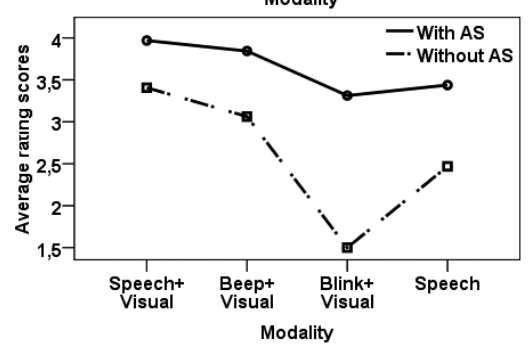

Modality

(d)

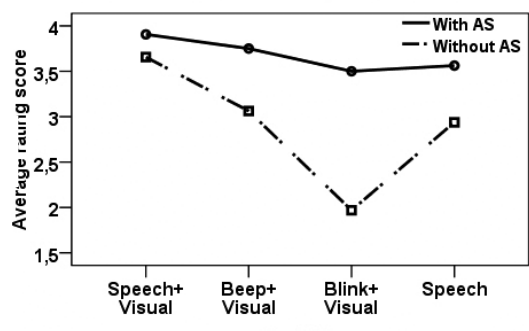

Modality

(e)

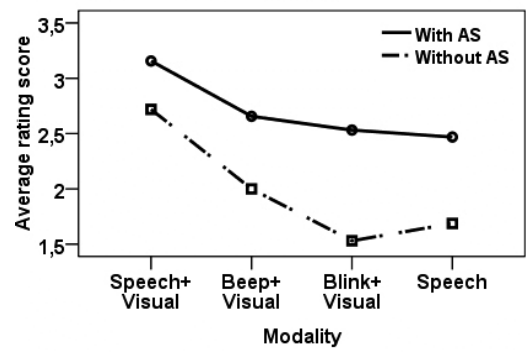

Figure 2. Average usefulness rating scores in each driving condition. (a) rich sound, (b) low visibility, (c) fatigue, (d) long drive, (e) high demand.

Long drive: The ratings in this condition closely resemble the ones in the 'fatigue' condition (Figure 2(d)). 
This might be due to the fact that a long drive on the highway often makes the driver feel tired and less attentive. The 'speech + visual' and the 'beep + visual' variants were judged as significantly more useful than the other two. The interaction effect was also present.

High demand: When the driving task imposes a high cognitive load on the driver, the freedom of perception becomes particularly important, because when available cognitive recourses are limited, drivers might need to frequently switch between the driving task and the warning perception/comprehension. The rating scores for this situation showed that the 'speech + visual' modality variant clearly stood out (see Figure 2(e)). According to Bonferroni tests, it was rated significantly higher than each of the other three variants, among which no significant difference was found. Auditory warnings can be perceived while keeping the eyes on the traffic, while visual warnings are self-paced and allow being read in segments at multiple times. In combination, the 'speech + visual' modality variant could provide the largest freedom of perception among the four.

In summary, visual modalities are highly necessary when driving with rich surrounding sounds. Auditory modalities are recommended in a low-visibility situation due to their "eyes-free" nature. Due to the ability to attract attention and enhance vigilance, auditory modalities are also suitable when the driver is tired or unconcentrated, or the trip is long and boring. The combination of visual and speech warnings offers freedom of perception, thus is particularly suitable during a highly demanding drive. In fact, this combination was rated as the (or one of the) most useful modality variant(s) in all five situations. However, this doesn't simply imply that local danger warnings should always be communicated redundantly via both visual modalities and speech. There are factors other than driving situation that might influence the selection of an appropriate communication mode, such as the type of information and the availability of system resources. Therefore, what is really needed for the design of adaptive systems is knowledge on how driving situation changes the requirements on communication modes, from which the appropriateness of each communication mode in each situation can be inferred.

\section{Conclusions and future work}

The situation-dependent usability assessment in this study has confirmed that it is indeed necessary for ADAS to adapt the communication mode of local danger warnings to changes in driving situation. First of all, the functionality of local danger warning might be more useful (appreciated) in some situations than in others. Drivers in this study considered local danger warnings the most useful when the visibility was low and the least useful when driving itself was highly demanding. Second, the driving situation also influences the requirements placed on communication modes. In this study, this influence was mainly reflected by the use of modality, rather than the level of assistance. Although derived from a local danger warning scenario, findings of this study can be applied to the design of ADAS in general. Future work is to obtain a deeper understanding of the interaction between communication mode and driving situation by investigating a wider range of ADAS functions, communication factors and situations. It would also be helpful to actually simulate the investigated situations in an experiment.

\section{Acknowledgements}

This work was funded by the EC Artemis project on Human-Centric Design of Embedded Systems (SmarcoS, Nr. 100249) and the German Ministry of Education and Research (project Car-Oriented Multimodal Interface Architecture, grant number 01IW08004).

\section{References}

[1] Brookhuis, K.A., D. De Waard, and W.H. Janssen. "Behavioural impacts of Advanced Driver Assistance Systemsan overview," European Journal on Transportation and Infrastructure Research, vol. 1, pp. 245-253, 2001.

[2] Kosch, T. "Local danger warning based on vehicle ad-hoc networks: Prototype and simulation," in First International Workshop on Intelligent Transportation (WIT'04), 2004.

[3] Reeves, L.M., J. Lai, J. A. Larson, S. Oviatt, T. S. Balaji, S. Buisine, P. Collings, P. Cohen, B. Kraal, and J.C. Martin. "Guidelines for multimodal user interface design," Communications of the ACM, vol. 47, pp. 57-59, 2004.

[4] Sarter, N.B. "Multimodal information presentation: Design guidance and research challenges," International Journal of Industrial Ergonomics, vol. 36, pp. 439-445, 2006.

[5] "ISO 9241: Ergonomic requirements for office work with visual display terminals (VDTS) - part 11: Guidance on usability," 1998.

[6] Cao, Y., A. Mahr, S. Castronovo, M. Theune, C. Stahl, and C. Müller. "Local danger warnings for drivers: The effect of modality and level of assistance on driver reaction," in International Conference on Intelligent User Interfaces (IUI'10), pp. $239-248,2010$.

[7] Bernsen, N.O. "Multimodality in language and speech systems - from theory to design support tool," in Multimodality in language and speech systems, B. Granström, D. House, and I. Karlsson, Eds., pp. 93-148, 2001.

[8] Spence, C., M.E.R. Nicholls, and J. Driver. "The cost of expecting events in the wrong sensory modality," Perception and Psychophysics, vol. 63, pp. 330-336, 2001. 